\title{
Cooperation between the human T-cell leukemia virus type-1 p30(II) protein and host cellular factors during oncogenic transformation and retroviral carcinogenesis
}

\author{
Megan Romeo*, Braden Barnett, Janice Kim, Paige Seeker, Jeffrey He, Alexander McRae, Fahmida Musharof, \\ Hayley Heatley, Afrida Faria, Jennifer Tran, Jordan Milam, Robert Harrod
}

From 16th International Conference on Human Retroviruses: HTLV and Related Viruses

Montreal, Canada. 26-30 June 2013

The human T-cell leukemia virus type-1 (HTLV-1) pXencoded accessory factors, p30II and Hbz, suppress proviral gene expression and help to maintain latency as a prerequisite for the development of adult T-cell leukemia/lymphoma (ATLL). Our laboratory is studying how these viral proteins influence host cellular gene expression and signaling which may contribute to hematological disease progression. There are five clinically-defined stages of HTLV-1-associated disease (pre-ATLL, smoldering T-cell leukemia, chronic T-cell leukemia, acute T-cell leukemia, and non-Hodgkin's T-cell lymphoma) and the transitional molecular events which lead to acute/lymphoma-stage disease are not well understood. We have previously shown that $\mathrm{p} 30^{\mathrm{II}}$ interacts with the c-MYC oncoprotein and enhances c-MYC-dependent transactivation and oncogenic potential by stabilizing recruitment of the TIP60 acetyltransferase to $\mathrm{p} 30^{\mathrm{II}} /$ c-MYC/TIP60 transcription complexes. We now extend these findings by demonstrating that $\mathrm{p} 30^{\mathrm{II}}$ induces acetylation of the c-MYC protein. Acetylation-defective Lys $\rightarrow>$ Arg substitution mutants of c-MYC (R5, K323R/K417R) are impaired for oncogenic foci-formation by $\mathrm{p} 30^{\mathrm{II}} /$ c-MYC. Acute/lymphoma-stage ATLL clinical isolates frequently exhibit overexpression of c-MYC, due to 8q24 chromosomal translocations and/or c-myc gene amplification, and the HTLV-1-transformed T-cell-lines HuT-102 and MJG11 display significant acetylation of c-MYC. The $p 53$ tumor suppressor is a downstream target of c-MYC

\footnotetext{
* Correspondence: mromeo@smu.edu

Laboratory of Molecular Virology, Department of Biological Sciences and the Dedman College Center for Drug Discovery, Design \& Delivery, Southern Methodist University, Dallas, Texas, USA
} 